Case Report

\title{
A Rare Case of Digital Ischemia and Gangrene in ANCA-Associated Vasculitis with Review of the Literature
}

\author{
Richard A. Lau, ${ }^{1}$ Ramandeep Bains, ${ }^{1}$ Duminda Suraweera, ${ }^{2}$ Jane $\mathrm{Ma}^{2}$ \\ Emil R. Heinze, ${ }^{1,2}$ Andrew L. Wong, ${ }^{1,2}$ and Philip J. Clements ${ }^{1}$ \\ ${ }^{1}$ UCLA-Olive View Rheumatology Program, Division of Rheumatology, Olive View-UCLA Medical Center, 14445 Olive View Drive, \\ 2B182, Sylmar, CA 91342, USA \\ ${ }^{2}$ UCLA-Olive View Internal Medicine Program, Department of Medicine, Olive View-UCLA Medical Center, 14445 Olive View Drive, \\ 2B182, Sylmar, CA 91342, USA
}

Correspondence should be addressed to Emil R. Heinze; emilheinze@gmail.com

Received 22 August 2016; Revised 19 October 2016; Accepted 24 January 2017; Published 28 February 2017

Academic Editor: Shigeko Inokuma

Copyright (C) 2017 Richard A. Lau et al. This is an open access article distributed under the Creative Commons Attribution License, which permits unrestricted use, distribution, and reproduction in any medium, provided the original work is properly cited.

\begin{abstract}
This paper describes one patient with Antineutrophil Cytoplasmic Antibody- (ANCA-) associated vasculitis who initially presented with multiple ischemic fingers and toes. On further evaluation, the patient was also found to have pulmonary-renal involvement and episcleritis. The diagnosis was supported with a positive cANCA (anti-proteinase 3) and a bronchoscopy consistent with diffuse alveolar hemorrhage. Although the patient refused a tissue biopsy, clinical presentation including nasal ulceration, sinus congestion, and epistaxis and anti-proteinase 3 antibody were more consistent with Granulomatosis with Polyangiitis (GPA) rather than Microscopic Polyangiitis (MPA) or Eosinophilic Granulomatosis with Polyangiitis (EGPA) based on the recently presented ACR/EULAR Provisional 2017 Classification Criteria for GPA (Luqmani et al., 2016). The patient responded well to therapy including high dose steroids and cyclophosphamide, with improvement of all organs involved and had no further digital ischemia or gangrene on follow-up. We include a review of the English literature summarizing presentation, management, and outcome of 16 similar cases.
\end{abstract}

\section{Introduction}

Antineutrophil Cytoplasmic Antibody- (ANCA-) associated vasculitis is a group of autoimmune small to medium vessel necrotizing vasculitides [1-6]. Granulomatosis with Polyangiitis (GPA), previously known as Wegener's, is a type of ANCA vasculitis that is usually associated with cANCA. The range of clinical manifestations of GPA can involve almost any organ system, but the classic organ systems involved include the upper respiratory tract, lower respiratory tract, and kidneys [1-6]. One of the rarer manifestations is digital ischemia and gangrene. We report a rare case of GPA presenting with digital ischemia and gangrene, with a review of the literature.

\section{Case}

A 62-year-old male with a past medical history of hypertension and vitiligo presented to the hospital with 2 months of worsening left 1st toe, left 3rd finger, and right 2nd, 3rd, and 4 th finger pain, swelling, and discoloration. The patient was a mechanic and endorsed an inciting traumatic event from dropping a heavy tool to his left 3rd finger and left 1st toe but denied any preceding trauma to the digits on his right hand. Soon after the initiation of the pain and swelling, there was also associated discoloration, with blue/black color changes with gradual scab formation.

On further review of systems, the patient reported several weeks of diffuse joint pains, intermittent eye pain and red eyes, hemoptysis, epistaxis, nasal congestion, and weight loss (unable to quantify but has decreased several belt notches). The patient otherwise denied any Raynaud's phenomenon, skin tightening, oral ulcerations, genital ulceration, chest pain, shortness of breath, abdominal pain, hematuria, ear pain, sinus pain, fevers, chills, night sweats, or smoking history.

The patient's initial vitals were significant for hypertension only. On physical exam, the patient was in no acute 


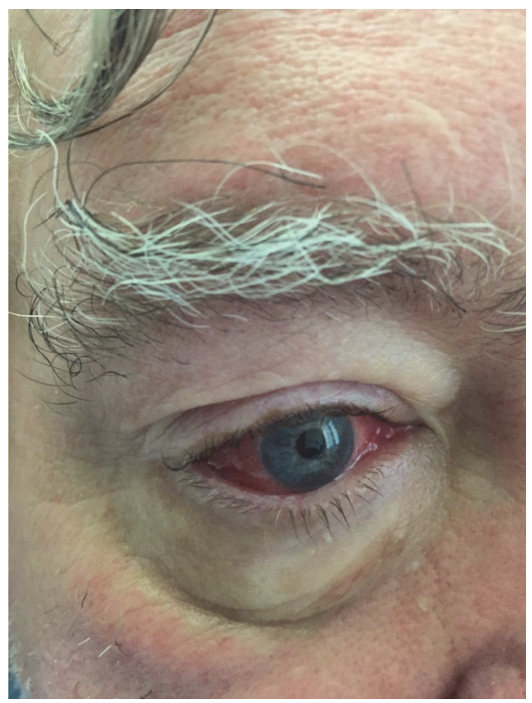

Figure 1

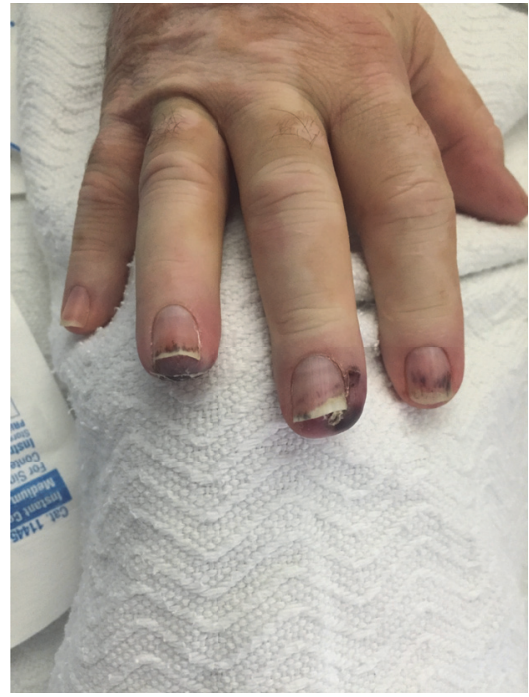

(a)

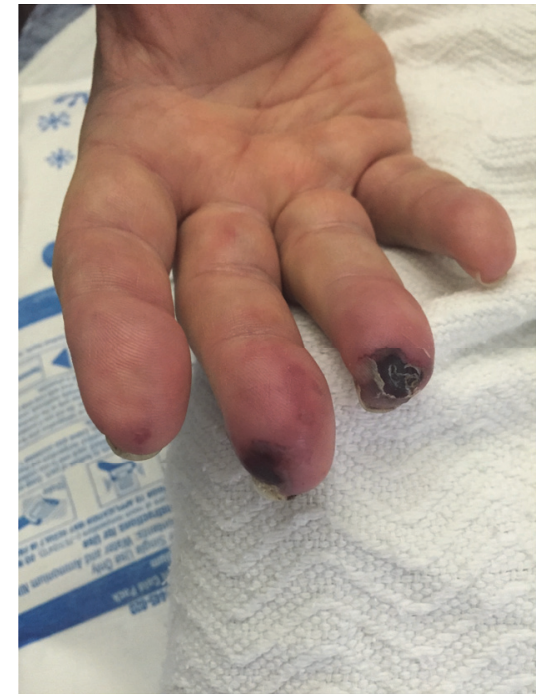

(b)

Figure 2

distress. The head and neck exam was notable for bilateral conjunctivitis (see Figure 1) and right nasal ulceration. The cardiopulmonary exam was notable for poor airflow on lung auscultation. The skin exam was notable for vitiligo. The musculoskeletal exam was significant for diffuse joint tenderness but no joint swelling/warmth. The extremity exam revealed $3+$ pitting edema of his lower extremities, right $2 \mathrm{nd} / 3 \mathrm{rd} / 4$ th finger duskiness and necrosis (see Figures 2(a) and 2(b)), left 3rd finger duskiness and necrosis (see Figures 3(a) and 3(b)), and left 1st toe with duskiness and necrosis (see Figures 4(a) and 4(b)).

The admitting labs were notable for elevated creatinine (1.74 mg/dL [ref 0.6-1.3]), elevated alkaline phosphatase
(180 units/L [ref 38-126]), elevated CRP (140 mg/L [ref 0.0$7.0])$, elevated ESR $(67 \mathrm{~mm} / \mathrm{hr}[\mathrm{ref}<20])$, leukocytosis $(12.5$ thousand cells/uL [ref 3.8-10.9]), and anemia (12.5 gm/dL [ref 13.6-17.3]). Urinalysis revealed the presence of large blood, red blood cells (113 cells/HPF [ref 0-3]), white blood cells (13 cells/HPF [ref 0-5]), protein (100 mg/dL [ref negative]), and cellular casts $(4 / \mathrm{LPF})$. Random urine protein/creatinine ratio was $0.959 \mathrm{gm} / \mathrm{gm}$. Initial chest $\mathrm{X}$-ray on admission showed patchy right upper lobe airspace disease.

Given both pulmonary and renal system involvement, there was concern for pulmonary-renal syndromes, including ANCA-associated vasculitides, antiglomerular basement membrane disease, systemic lupus erythematous, 


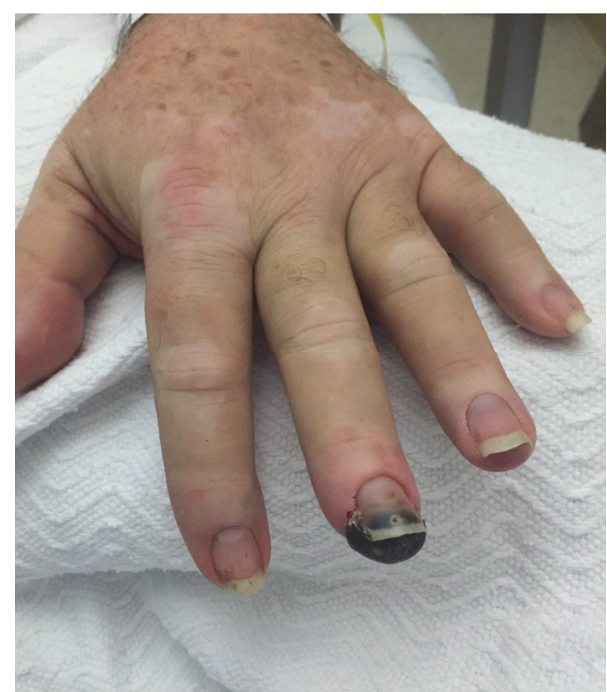

(a)

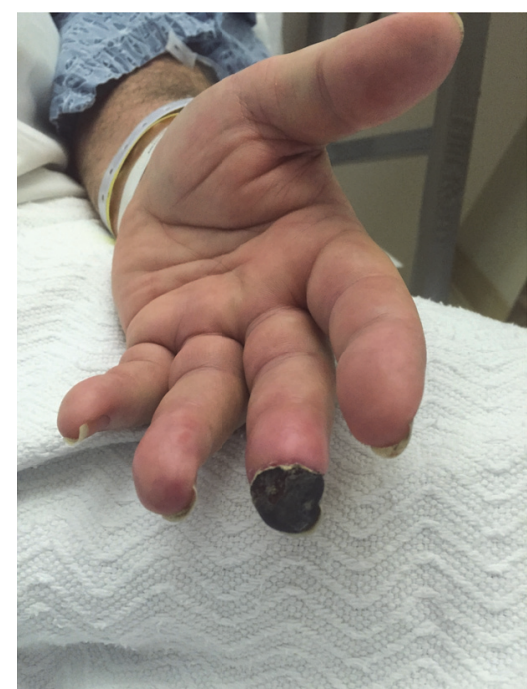

(b)

Figure 3

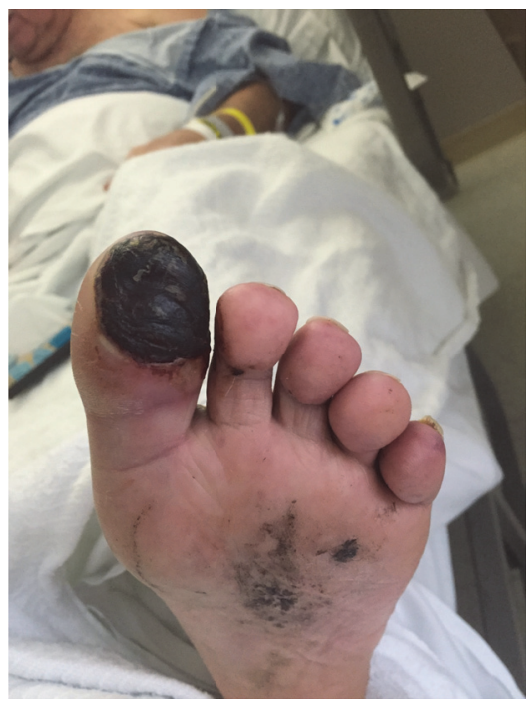

(a)

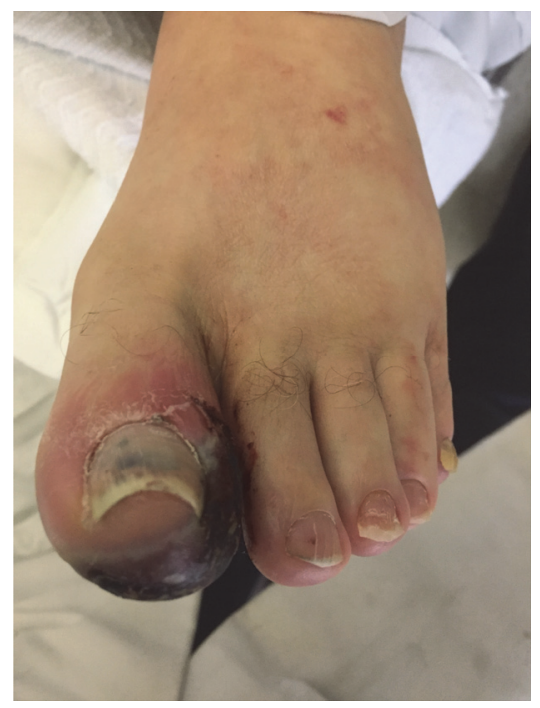

(b)

Figure 4

and cryoglobulinemia. Other vasculitides of consideration included polyarteritis nodosum and thromboangiitis obliterans. We also considered that prothrombotic disorders such as antiphospholipid syndrome and embolic phenomenon can occur with cholesterol emboli or atrial myxoma. Infectious etiologies were considered as well, including endocarditis with embolic phenomenon. A paraneoplastic syndrome from a malignancy was entertained as well.

Further investigation was pursued to workup the aforementioned etiologies, including more laboratory evaluation with serologies pertinent for positive c-ANCA (proteinase-3 Ab $3.1 \mathrm{AI}[\mathrm{ref}<1.0])$, positive rheumatoid factor $(80 \mathrm{IU} / \mathrm{mL}$ [ref < 14]), negative ANA, negative antiglomerular basement membrane antibody, negative anti-SSA/SSB, negative cryoglobulin screen, negative lupus anticoagulant, negative anti-Beta2 glycoprotein, and negative anticardiolipin. The infectious laboratory workup included multiple blood cultures with no growth. Transthoracic echocardiography did not show any thrombus, valvular disease, or atrial myxoma. Additional imaging was pursued that included computer tomography of the chest, which demonstrated extensive consolidation with air bronchograms predominately involving the right upper lobe and some contiguous involvement of the right middle and lower lobes. Bronchoscopy with serial 


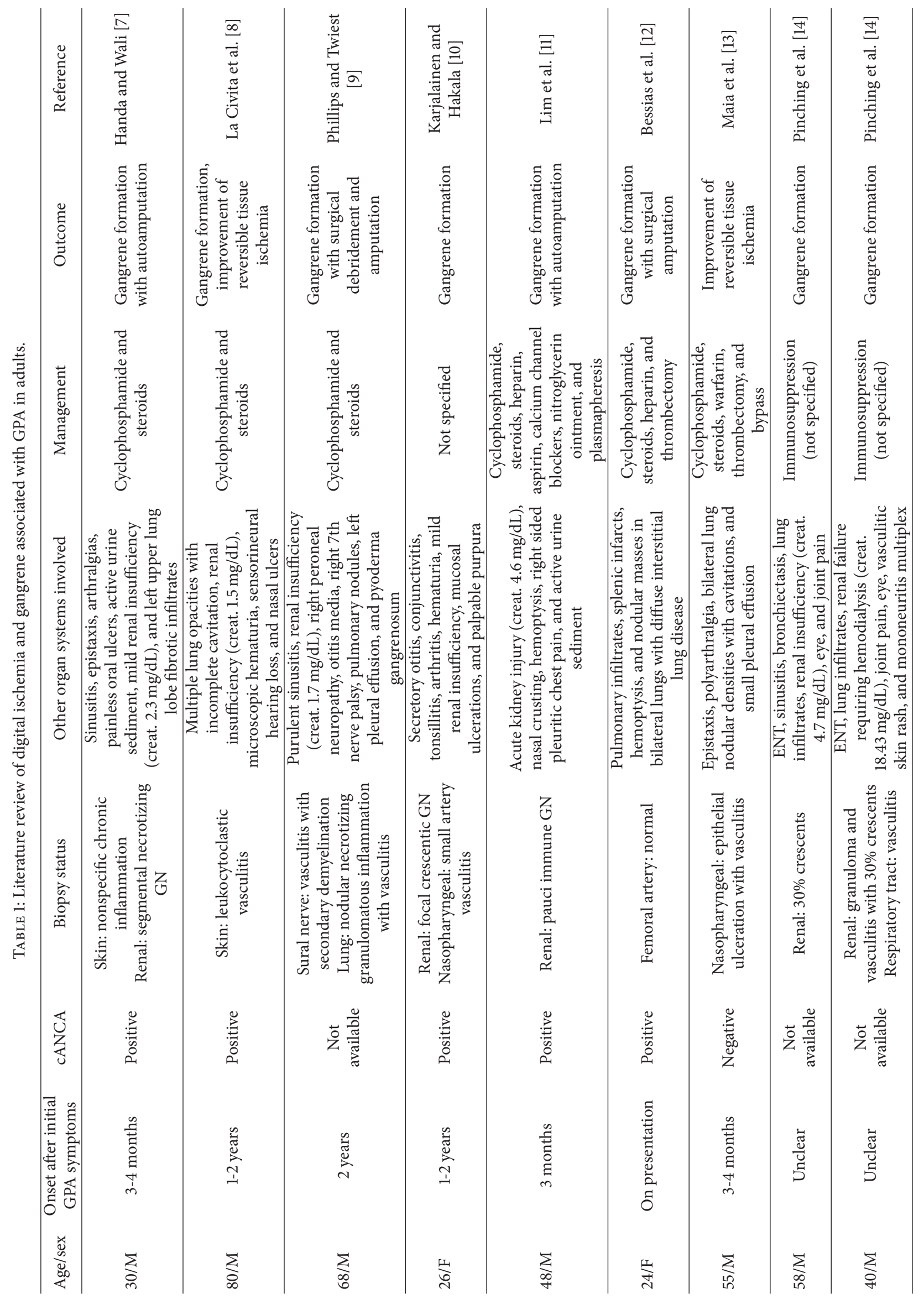




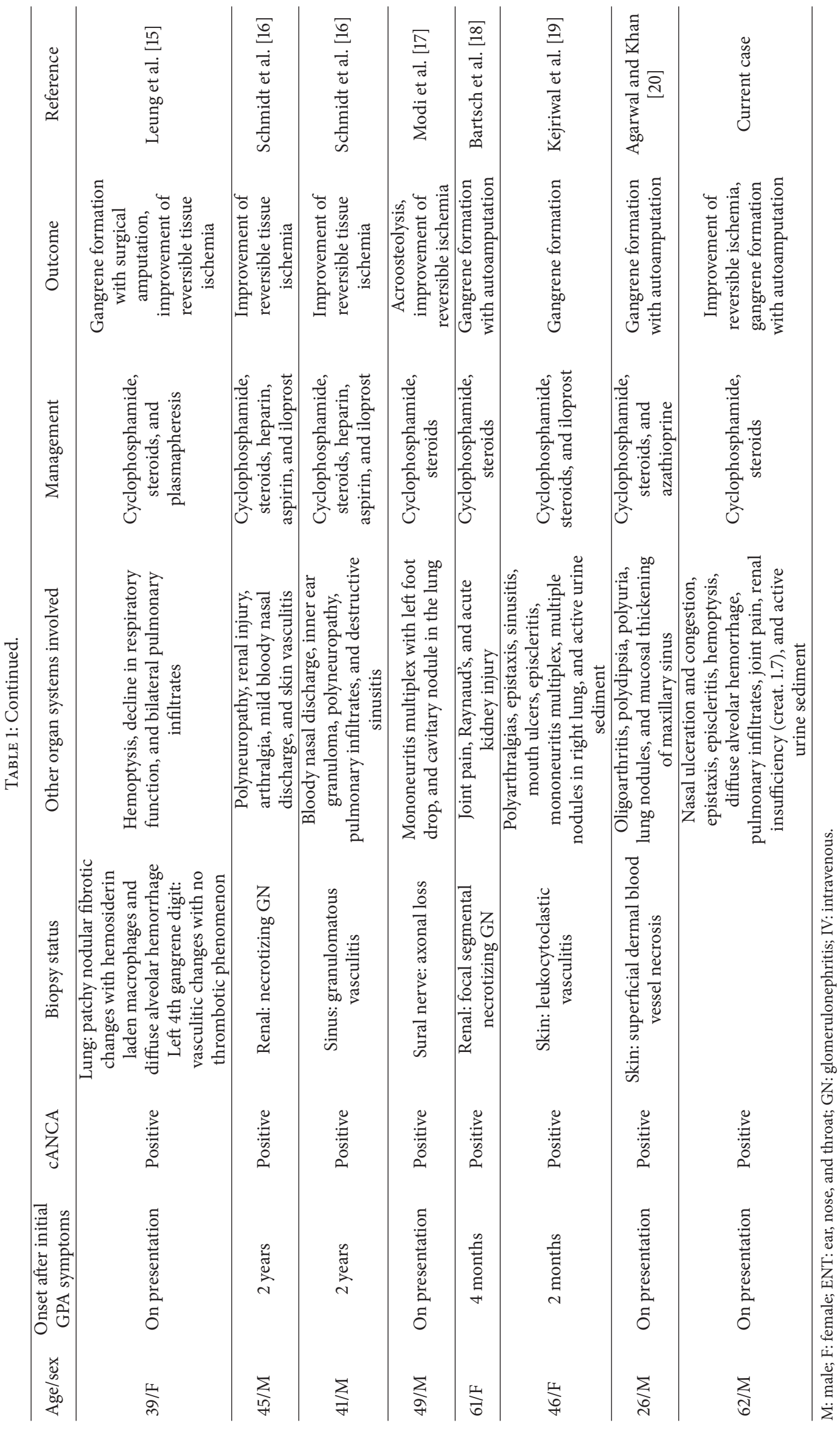


bronchial washings by pulmonary consultants was consistent with alveolar hemorrhage. The ocular examination by ophthalmology consultants was consistent with episcleritis.

The patient was diagnosed with ANCA-associated vasculitis. The defining pathological difference between Granulomatosis with Polyangiitis (GPA) and Microscopic Polyangiitis (MPA) is the presence of granulomatous changes on biopsy based on our current classification criteria [1-3]. To support the diagnosis, we were planning on a skin biopsy but the patient refused. A renal biopsy was scheduled but the patient did not show for the procedure. Often even if biopsies are performed, there is a risk of missing definitive granuloma tissue due to sampling error, which makes differentiating the type of ANCA-associated vasculitis difficult. Based on the recently presented ACR/EULAR Provisional 2017 Classification Criteria for GPA, the patient scores 4 points on clinical criteria given his bloody nasal discharge, sinonasal congestion, and red or painful eyes and scores 5 points on serologic criteria with a positive cANCA-PR3 antibody. A combined score of greater than or equal to 5 is needed for classification of GPA [21]. The patient was classified as most likely having GPA and treated with IV methylprednisolone followed by oral prednisone, which was slowly tapered over the next several months. He was also started on monthly IV cyclophosphamide for a total of 6 months. The patient responded well to therapy with improvement of all of his organ dysfunction, including improvement of the reversible tissue ischemia, cessation of digital gangrenous extension, and eventual autoamputation of the irreversible gangrenous parts of his digits.

\section{Discussion}

Based on our review of the English literature, to the best of our knowledge, only 16 cases have been previously reported describing adult patients with GPA who presented with digital ischemia and gangrene [7-20] (see Table 1). Given the paucity of cases reported, we suspect the prevalence with digital ischemia and gangrene in the GPA population to be $<1 \%$. All of the cases in this review, including our case, satisfy the ACR/EULAR Provisional 2017 Classification Criteria for GPA with the exception of the 58-year-old male, patient number 17, in the case series by Pinching et al. where not enough specific case details were reported to confirm the diagnosis. The mean age of patients was 46.9 years and included a range of 24-80 years. Including our patient, 12 of 17 were males (71\%) compared to 5 of 17 females (29\%). Most patients presented with multiorgan involvement including 14 of 17 with pulmonary involvement (82\%), 11 of 17 with kidney disease $(65 \%)$, and 13 of 17 with sinonasal disease (76\%). cANCA was confirmed positive in 13 patients and negative in one patient, and the data was not available in 3 of the cases. Only 3 patients demonstrated granulomas on biopsy. Confounding risk factors were inconsistently reported but include a past medical history of HTN in three cases $[9,16,19], \mathrm{DM}$ in three cases $[13,15,16]$, tobacco use in two cases $[13,18]$, and dyslipidemia in one case [13]. The clinical phenotype usually involves multiple disparate digits. 10 of 17 cases described isolated involvement of the upper extremities (59\%) compared to the 5 of 17 with isolated involvement of the lower extremities (29\%). Only two cases (12\%), including ours, have described simultaneous involvement of both the upper and lower extremities [20]. The pathophysiology of the digital ischemia and gangrene is thought to be from destruction of medium sized vessels from active vasculitis, which has been demonstrated in case reports where histological examination of affected tissue showed arteritis $[9,15]$. However, there is also evidence that in situ thrombosis, as a result of active vasculitis, can lead to ischemia and gangrene, as seen in angiographic examination of affected patients, as well as on histological examination of affected tissue showing arterial thrombi [12, 13]. Initial symptoms in most cases included pain and swelling with eventual gangrene formation if left untreated. Digital ischemia and gangrene can be part of the initial presentation of GPA or develop later in the course of the disease, even while the patient is on active treatment. The diagnosis is usually made based on clinical grounds, but angiographic examination can support vasculitic or thrombotic lesions [720]. Pathology can demonstrate either vascular arteritis or thrombi formation $[9,12,13,15]$. There is no consensus on treatment regarding GPA with digital vasculitis with ischemia and gangrene. However, cyclophosphamide and steroids are the most commonly used treatment in the literature [7-20]. Other therapies that have been used include anticoagulation and vasodilators. Surgical intervention with thrombectomy and bypass have been used as well.

Digital ischemia and gangrene are rare manifestations of GPA. They can have a heterogeneous presentation as demonstrated by the cases in the literature. Diagnosis can be difficult as biopsy of the skin is often nonspecific. Without treatment, ischemia and gangrene can progress and lead to significant disability. Therefore, it is important for clinicians to be aware of this rare manifestation and institute early treatment as indicated.

\section{Competing Interests}

The authors declare that there is no conflict of interests regarding the publication of this paper.

\section{References}

[1] J. C. Jennette and R. J. Falk, "Small-vessel vasculitis," New England Journal of Medicine, vol. 337, no. 21, pp. 1512-1523, 1997.

[2] C. O. S. Savage, L. Harper, and D. Adu, "Primary systemic vasculitis," Lancet, vol. 349, no. 9051, pp. 553-558, 1997.

[3] P. M. K. Lutalo and D. P. D'Cruz, "Diagnosis and classification of granulomatosis with polyangiitis (aka Wegener's granulomatosis)," Journal of Autoimmunity, vol. 48-49, pp. 94-98, 2014.

[4] P. Lamprecht and W. L. Gross, "Wegener's Granulomatosis," Herz, vol. 29, no. 1, pp. 47-56, 2004.

[5] A. D. Mahr, T. Neogi, and P. A. Merkel, "Epidemiology of Wegener's granulomatosis: lessons from descriptive studies and analyses of genetic and environmental risk determinants," Clinical and Experimental Rheumatology, vol. 24, no. 2, supplement 41, pp. S82-S91, 2006. 
[6] S. West, N. Arulkumaran, P. W. Ind, and C. D. Pusey, "Diffuse alveolar haemorrhage in ANCA-associated vasculitis," Internal Medicine, vol. 52, no. 1, pp. 5-13, 2013.

[7] R. Handa and J. P. Wali, "Wegener's granulomatosis with gangrene of toes," Scandinavian Journal of Rheumatology, vol. 25, no. 2, pp. 103-104, 1996.

[8] L. La Civita, G. Jeracitano, C. Ferri, R. Pedrinelli, G. Dell'Omo, and G. Catapano, "Wegener's granulomatosis of the elderly: a case report of uncommon severe gangrene of the feet," Annals of the Rheumatic Diseases, vol. 54, no. 4, 1995.

[9] R. W. Phillips and M. W. Twiest, "Wegener's granulomatosis and gangrene in the feet," Annals of Internal Medicine, vol. 99, no. 4, p. 571, 1983.

[10] A. Karjalainen and M. Hakala, "Still another case of Wegener's granulomatosis with a digital gangrene," Scandinavian journal of rheumatology, vol. 25, no. 5, p. 339, 1996.

[11] S. Y. Lim, J. H. Lim, and C. Horn, "Upper-extremity digital ischemia in granulomatosis with polyangiitis," Journal of Clinical Rheumatology, vol. 20, no. 3, pp. 155-159, 2014.

[12] N. Bessias, K. G. Moulakakis, C. Lioupis et al., "Wegener's granulomatosis presenting during pregnancy with acute limb ischemia," Journal of Vascular Surgery, vol. 42, no. 4, pp. 800804, 2005.

[13] M. Maia, P. Brandão, P. Monteiro et al., "Upper limb ischemia in a patient with Wegener's granulomatosis," Interactive Cardiovascular and Thoracic Surgery, vol. 7, no. 6, pp. 1137-1140, 2008.

[14] A. J. Pinching, C. M. Lockwood, B. A. Pussell et al., "Wegener's granulomatosis: observations on 18 patients with severe renal disease," Quarterly Journal of Medicine, vol. 52, no. 208, pp. 435460, 1983.

[15] A. Leung, C. B. Sung, G. Kothari, C. Mack, and C. Fong, "Utilisation of plasma exchange in the treatment of digital infarcts in Wegener's granulomatosis," International Journal of Rheumatic Diseases, vol. 13, no. 4, pp. e59-e61, 2010.

[16] W. A. Schmidt, D. Wernicke, E. Kiefer, and E. Gromnica-Ihle, "Colour duplex sonography of finger arteries in vasculitis and in systemic sclerosis," Annals of the Rheumatic Diseases, vol. 65, no. 2, pp. 265-267, 2006.

[17] M. Modi, A. K. Vats, S. Prabhakar, V. Singla, and S. Mishra, "Acro-osteolysis and mononeuritis multiplex as a presenting symptom of systemic angiitis of Wegener's type," Indian Journal of Medical Sciences, vol. 61, no. 4, pp. 212-215, 2007.

[18] P. Bartsch, A. Schossee, J. Sitte, P. R. Mertens, and G. H. Neild, "Joint pain, digital necrosis and acute kidney failure in smallvessel autoimmune disease," Clinical Kidney Journal, vol. 6, no. 2, pp. 240-242, 2013.

[19] R. Kejriwal, T. M. Lim, and S. Kumar, "Wegener's granulomatosis and acute upper limb digital ischemia," International Journal of Rheumatic Diseases, vol. 11, no. 2, pp. 185-187, 2008.

[20] A. Agarwal and N. A. Khan, "Multiple digital gangrene and polyuria," Mayo Clinic Proceedings, vol. 87, no. 4, article e25, 2012.

[21] R. Luqmani, P. A. Merkel, and R. Watts, "New classification criteria for ANCA-associated vasculitis: implications for clinical practice," in Proceedings of the ACR/ARHP Annual Meeting, J. Robson, Ed., Washington, DC, USA, November 2016. 


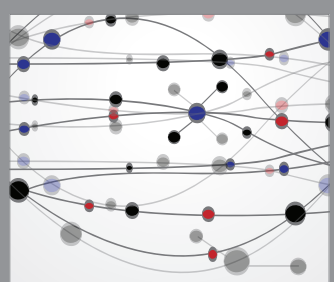

The Scientific World Journal
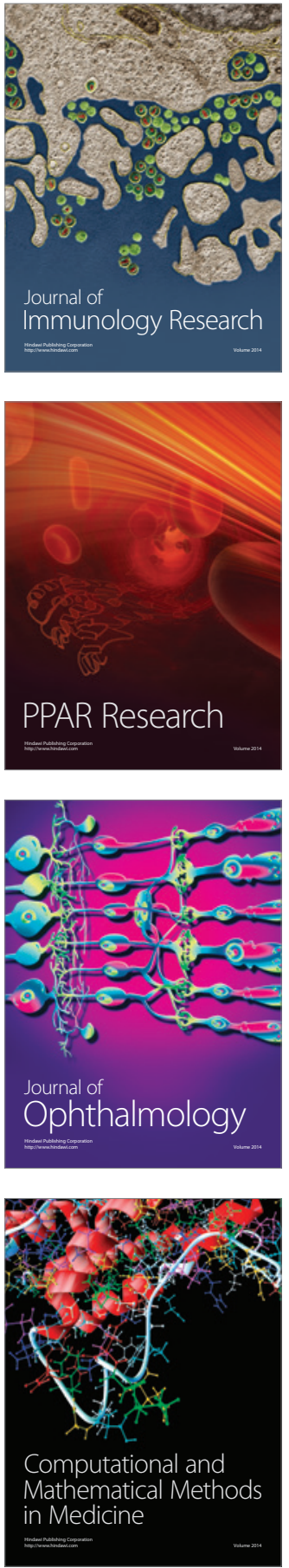

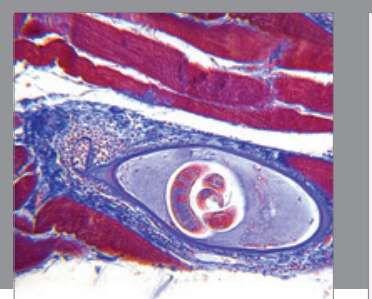

Gastroenterology Research and Practice
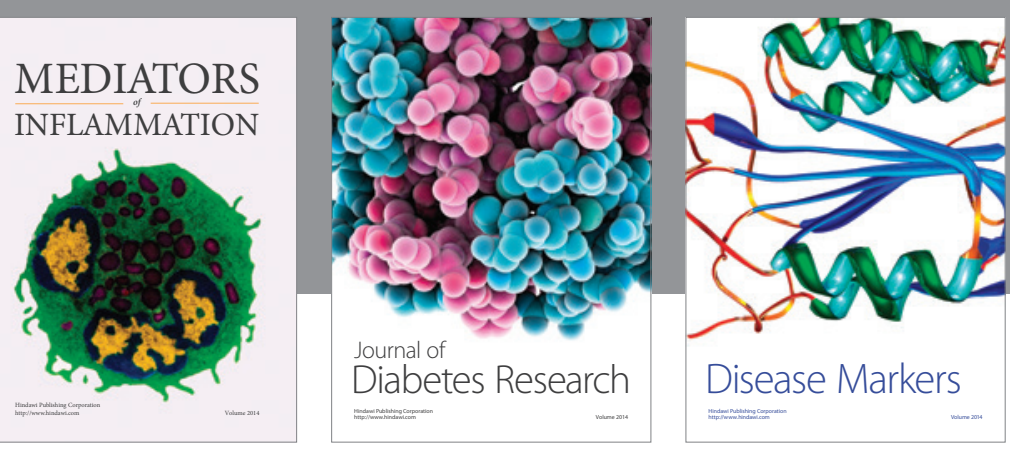

Disease Markers

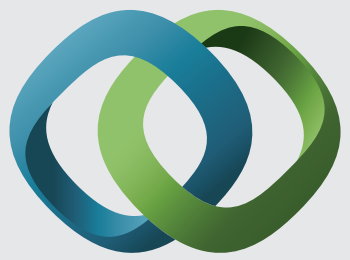

\section{Hindawi}

Submit your manuscripts at

https://www.hindawi.com
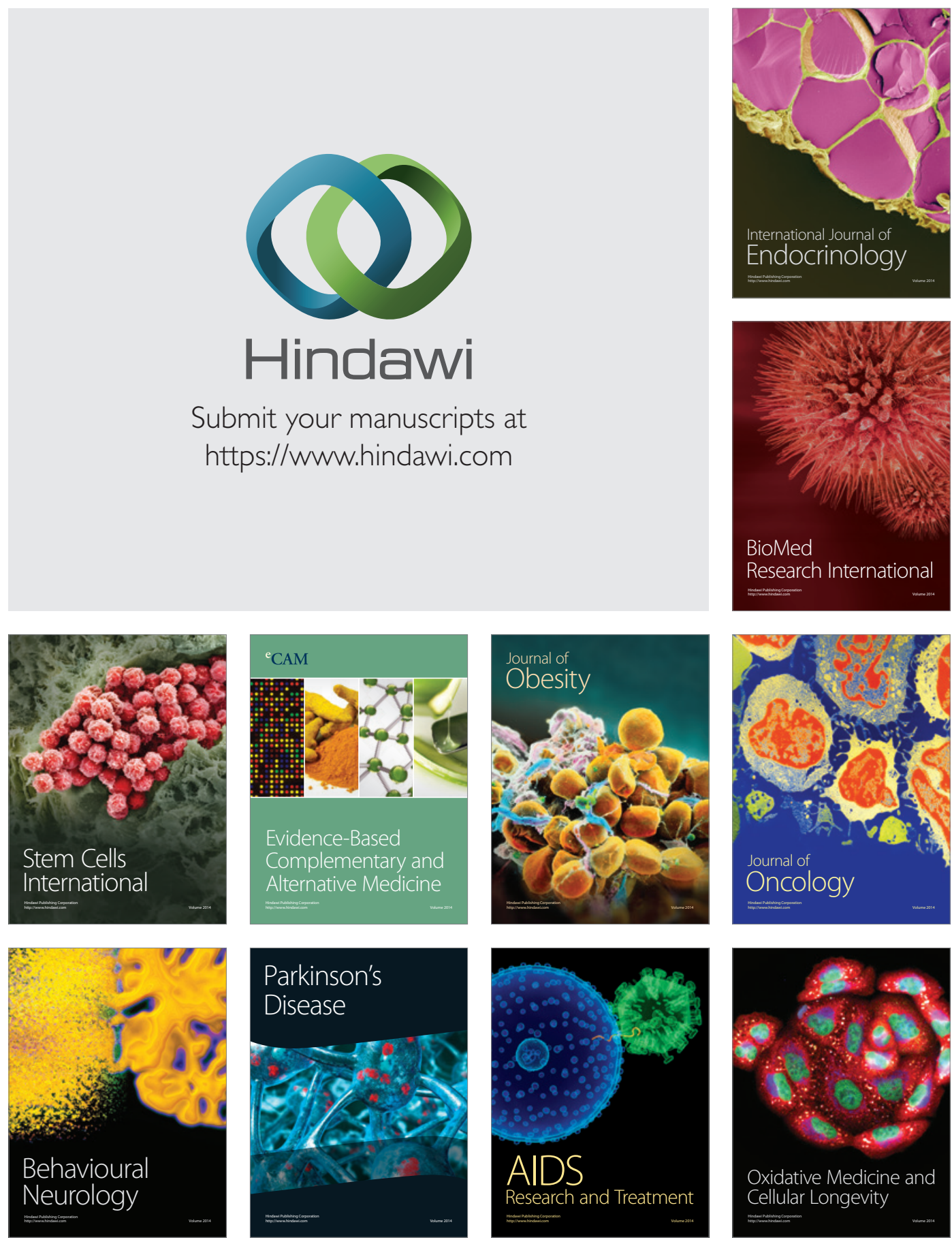